\title{
Angularectopicpregnancy: About a Case Study at the Teaching and Department Hospital of Borgou Alibori
}

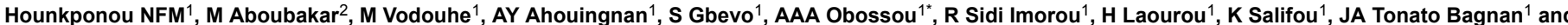
RX Perrin ${ }^{2}$

${ }^{1}$ Department of Mother and Child, Faculty of Medicine, University of Parakou, Benin

${ }^{2}$ Faculty of Health Sciences, University of Abomey-Calavi, Benin

${ }^{*}$ Corresponding author: Awadé Afoukou Achille Obossou, Obstetrician-Gynecologist, Faculty of Medicine, University of Parakou, Benin, Tel: (229) $95853279 / 970678$ 52; E-mail: awadefr2000@yahoo.fr

Received date: June 06, 2017; Accepted date: July 15, 2017; Published date: July 25, 2017

Copyright: @2017 Hounkponou NFM, et al. This is an open-access article distributed under the terms of the Creative Commons Attribution License, which permits unrestricted use, distribution, and reproduction in any medium, provided the original author and source are credited.

\begin{abstract}
The authors report a case of angular ectopic pregnancy interrupted at six weeks of amenorrhoea with the patients of 32 years old, with her $2^{\text {nd }}$ pregnancy, nulliparous an antecedent of laparotomy for right ectopic pregnancy (EP). At the admission, the clinical examination and the ultrasound have permitted to make a diagnosis of an abdominal pregnancy. During the operation the diagnosis of a left retort ectopic pregnancy has been made. It has profited from a suture with a perfect haemostasis. The aftermaths of the operation have been simple and the patient has been recovered five days (5 days) after the operation.
\end{abstract}

Keywords: Angular ectopic pregnancy; Fœtal death; Benin

\section{Introduction}

Defined as the implantation of the embryo in the horn of the uterus, the pregnancy in the horn of the uterus is a rare entity and represents about $2 \%$ of the ectopic pregnancies (EP) [1]. The diagnosis of the angular or interstitial remains difficult. The interruption remains a mode of frequent revelation for this type of ectopic pregnancy [2]. The angular pregnancies are dangerous and their rate of mortality oscillates between $2 \%$ and $5 \%$ [2]. The agreement to bear the medical cost of the angular pregnancies is often codified, and often guided by the clinic chart [3].

\section{Observation}

It was about madam C.A. aged of 25 second and nulliparous pregnancy, 0 living child, referred to the maternity of the Departmental and University hospital Centre of Borgou and Alibori at parakou in Benin because of bleeding on pregnancy of undetermined term. The familial antecedents were without particularities. The individual antecedents were essentially marked by a laparotomy two years ago for an ectopic pregnancy. The operating protocol has not been viewed. The clinical test reveals a total altered, the blade palpebral mucous membrane, the anicteric bulb mucous membranes, the temperature at $37^{\circ} \mathrm{C}$, the pulse at 110 pulsations/min and the blood pressure at $80 / 50 \mathrm{mmHg}$.

The urinary strip is tested negative. The breasts are normal, the abdomen that amounted in volume and painful has a big longitudinal axis with a median scar of laparotomy. The uterus is difficult to exam with the sensation of a young fotus and a scream of the naval. The achieved ultrasound has shown a mono foetal pregnancy non evolutionary of 28 weeks of amenorrhoea plus one day in the abdomen with an empty uterus and an abdominal outpouring. The annexes are hard to appreciate. The diagnosis of the abdominal pregnancy with a shocking state has been then raised. It has benefited from the preparation with a double venous board followed by vascular filling. An emergency check-up has been made of Rhesus Blood Group (RBG): O positive, haemoglobin rate at $7 \mathrm{~g}$, uraemia $0.4 \mathrm{~g} / \mathrm{l}$ and creatininmy $12 \mathrm{mg} / \mathrm{l}$. A blood transfusion (a sac) has been conducted before the operating theatre suite. It has benefited from a laparotomy.

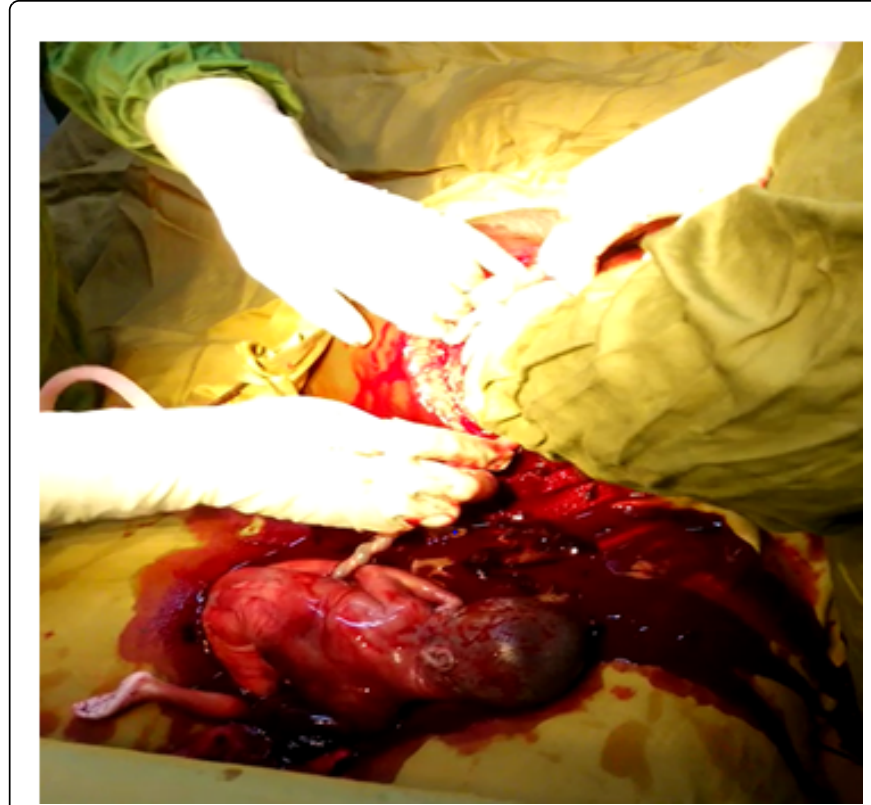

Figure 1: Fresh still-born fœtus.

At the coeliotomy appears a haemoperitoinia that one sucks up followed by the extraction of a fresh still-born foetus of the abdomen (Figure 1). The exploration of the pelvic cavity reveals a placenta insertion in the angle of the uterine that one removes followed by cleaning. One proceeds by sucking up the ovular debris of the angle and the uterine cavity. One achieves a historiography with a correct 
Citation: Hounkponou NFM, M Aboubakar, M Vodouhe, AY Ahouingnan, S Gbevo, et al. (2017) Angularectopicpregnancy: About a Case Study at the Teaching and Department Hospital of Borgou Alibori. Clinics Mother Child Health 14: 266. doi:10.4172/2090-7214.1000266

Page 2 of 3

haemostasis. The exploration of the annexes reveals a salpingectomy right and an inflammatory left horn but with normal aspect (Figure 2). The left ovary is also normal. The cleaning of the abdominal cavity and the closing of the wall plan by plan has been achieved. Two sacks of blood have been transfused her before after the surgical operation. The operating theatre suites have been simple and the patient is released five days after the operation with a test of haemoglobin at $10 \mathrm{~g} / \mathrm{dl}$.

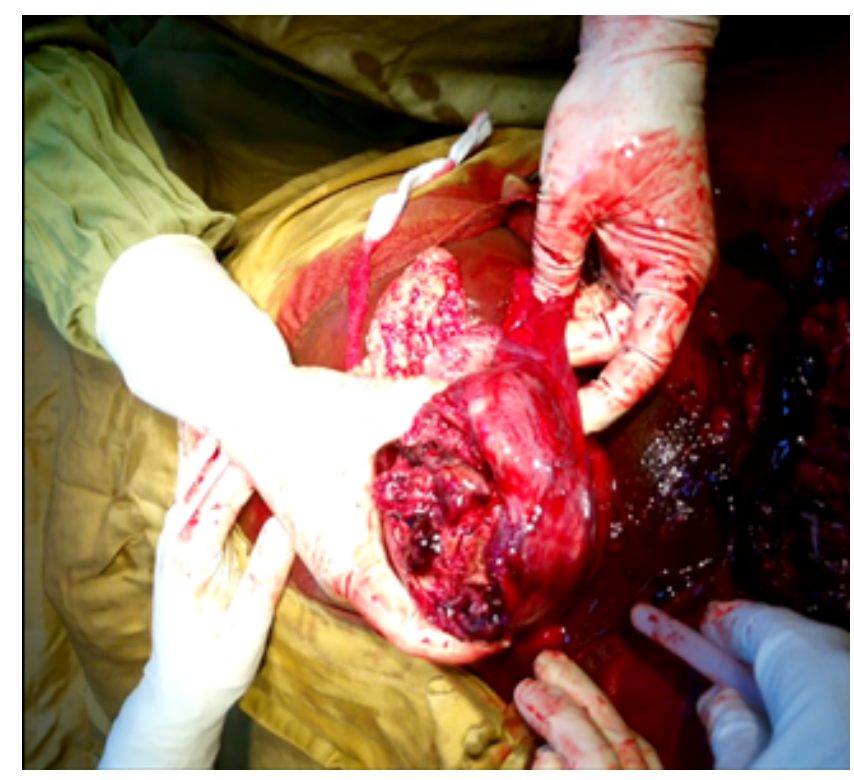

Figure 2: Left horn.

\section{Discussion}

The angular pregnancy is a rare entity which is distinguished from the other types of ectopic pregnancy by its greatest haemorrhage risk with a larger indication of radical treatment [1], the signalled case in our study is 25 years old with characteristics of paucigest, nulliparous and 0 living child. Similar studies underscore descriptions enough close to ours. Driisi et al. [1] report a case aged of 27, second, primipara, and mother of a living child. A case aged of 28 has been reported by Ferjaoui et al. in Tunisia [4]. At CHU in Yopougon a patient aged of 27 and nulliparous has been signalled by Kouame et al. In Turkey a particular case of association ectopic pregnancy and ectopic pregnancy with eleven weeks of amenorrhoea has been described with a patient of 32 years old $[5,6]$. One can then conclude that an angular pregnancy is an abnormality from the young women. The pelvic pains are motives of admission common in the studies of Drisi and Ferjaoui [1,4]. Moreover, the factors of common risks of ectopic pregnancies (advanced maternal age, tobacco, contraception by micro progestin, endometriosis and pelvic surgical antecedents, high recurring genital infections), the angular localization to these specific factors that is to say: the uterine malformations with rudimentary horn and the antecedents of salpingectomy with angular pregnancy on the uterine stump [1].

Contrary to our study that has not raised the diagnosis of abdominal pregnancy with a state of shock in the first time, that one by Kouame [5] and by Peker [6] have directly raised the diagnosis of an angular pregnancy on the data of the ultrasound and the hysterosalpingography. Their diagnosis has been confirmed by the dosage of
beta-HCG (6000 mUI/mL) [5]. What leads some authors conclude that the diagnosis of angular pregnancy is difficult. The diagnosis of angular pregnancy is often by default by sub-pubic or transparietal ultrasound $[1,5]$. In effect, the gestational sack can appear intrauterine fundic then delaying the diagnosis at the level of the interruption engaging the vital prognosis of the woman [1]. In its typical form, to the endovaginal ultrasound, the uterus appears empty [1]. This assertion has been confirmed in the studies by Ferjaoui [4] and Kouame [5]. In our study we have come across an empty uterus with abdominal outpouring. The gestational sac when it is visible achieves transferable fundic surrounded by myometer. However, the horn seat is often discovered during the operation [1]. What reveals the agreement to pay the medical expenses, as in our study, the portrayed patient in the one by Kouame [5]. The radical treatment by salpingectomy with horn resection is the classical treatment. The medical treatment by methotrexates in situ seems to be an interesting therapeutic alternative when it is possible.

The selective embolization has been recently suggested as efficient treatment. Presently apart from the situation of interruption, the treatment by injections, possibly iterative, methotrexates by systemic canal or in situ coupled with a monitoring of the kinetic of plasmatic beta-HCG and a following up by ultrasound are often implemented in the intention [1]. In a retrospective study (206-2015) conducted by Nikodijevic [3] CHU Jean-Verdier in France, six (06) patients out of nineteen had been medically treated with methotrexates (one unsuccessful case has surgically) and 13 patients had benefited from a surgical treatment by the resection of the horn. The angular ectopic pregnancies (EP) are pregnancies with risk of haemorrhagic interruption at short term and recurring at medium-dated. A recent technique of agreement to medical expenses of the angular pregnancy has been described by Sergent in 2003 [2]. It is about the endoloop around the basis of the angular pregnancy. By traction of the thread of the endoloop, haemostasis is assured. The ectopic pregnancy can then be evacuated in safety. It is signalled that at the end of the procedure, the endoloop set up in maximum traction ensuring then a definitive haemostasis. In this case, the tub integrity cannot be respected.

This technique though simple rapid, has a potential drawback: that of the skid of the lasso, mainly when it is about voluminous angular pregnancies [2]. It is for this reason that the therapeutic choice has been guided chiefly by the clinic chart. Kouame [3] has underscored that the endo-vaginal ultrasound coupled with dosage of plasmatic beta-HCG permits the diagnosis at the stage of precocious angular pregnancy [5]. The prognosis of fertility and the risk of recurring depend on obviously on the state of control-lateral horn. As for the obstetrical prognosis, it has been marked by the risk of uterine rupture, so, the caesarean seems to be justified during a coming pregnancy [2]. Other researchers have reported some cases of ectopic pregnancy associated with intrauterine pregnancy normally progressed and led to a planned caesarean at 39 weeks of amenorrhoea [6]. Similar cases have been observed after an in vitro fecundation reported by Oral [7]. Whatever the treatment is, the prognosis of fertility and the ulterior obstetrical prognosis seem good underscores Nikodijevic, Peker and Oral $[3,6,7]$.

\section{Conclusion}

Angular pregnancy is a rare event and leads to unknown ectopic pregnancy. The maternal vital prognosis can be put in relief some fearsome haemorrhagic complications. A precocious diagnosis and an adequate agreement to pay medical expenses are necessary. This 
Citation: Hounkponou NFM, M Aboubakar, M Vodouhe, AY Ahouingnan, S Gbevo, et al. (2017) Angularectopicpregnancy: About a Case Study at the Teaching and Department Hospital of Borgou Alibori. Clinics Mother Child Health 14: 266. doi:10.4172/2090-7214.1000266

Page 3 of 3

observation raises the problem a bad following up of the pregnancies hence the interest of continuous sensitization of the population.

\section{References}

1. Drissi J, Fagouri H, Derdabi H, Kassidi F, Guelzim K, et al. (2015) Grossesse cornuale : à propos d'un cas et revue de la littérature. Int J Innovation Appl Studies 13: 168-171.

2. Sergent $\mathrm{F}$ (2003) Excision cornuale coelioscopique à la pince automatique des grossesses interstitielles rompues. J Gynécol Obst Biol Repro 32: 426-430.

3. Nikodijevic K, Bricou A, Benbara A, Moreaux G, Nguyen C, et al. (2016) Grossesse extra-utérine cornuale: Prise en charge, fertilité ultérieure et devenir obstétrical. Gynécol Obst Fertil 44: 11-16.
4. Ferajoui MA, Gharrad M (2016) La grossesse cornuale, aspect laparascopique. Pan African Med J 23: 245.

5. Kouame N, N'Goran-Domoua AM, Keita AK, N'Gbesso RD (2011) Grossesse cornuale: une entité rare de grossesse extra-utérine. Imagerie Femme 21: 68-71.

6. Peker N, Aydeniz EG, Gündoğan S, Şendağ F (2017) Laparoscopic Management of Heterotopic Istmocornual Pregnancy: A Different Technique. J Minim Invasive Gynecol 24: 8-9.

7. Oral S, Akpak YK, Karaca N, Babacan A, Savan K (2014) Cornual heterotopic pregnancy after bilateral salpingectomy and uterine septum resection resulting in term delivery of a healthy infant. Obstet Gynecol 3. 